\title{
Towards Examining The Effects of Live Streaming an Educational Game
}

\author{
Noor Hammad \\ nhammad@cs.cmu.edu \\ Carnegie Mellon University \\ Pittsburgh, Pennsylvania
}

\author{
Erik Harpstead \\ harpstead@cmu.edu \\ Carnegie Mellon University \\ Pittsburgh, Pennsylvania
}

\author{
Jessica Hammer \\ hammerj@andrew.cmu.edu \\ Carnegie Mellon University \\ Pittsburgh, Pennsylvania
}

\begin{abstract}
We propose a study on the effects of live streaming on an educational game's learning outcomes. The COVID-19 pandemic has strengthened the call for interactive online learning experiences. There is a growing body of literature examining learning on platforms such as Twitch, and studies have shown that enhancing in-game performance is possible from viewing a stream. However, little work has explored whether learning from live streaming educational games, where in-game performance relates to educational outcomes outside of the game context, is possible. We share the details of our proposed study, in which an educational game called Angle fungle will streamed to participants, and learning gains will be compared to three non-live streamed conditions. By executing this study, we can understand the benefits and shortcomings of current live streaming interfaces in supporting educational games, paving the way for the design of novel and interactive learning experiences built for live streaming platforms.
\end{abstract}

\section{CCS CONCEPTS}

- Human-centered computing $\rightarrow$ User studies; • Applied computing $\rightarrow$ Computer games; • Social and professional topics $\rightarrow$ K-12 education.

\section{KEYWORDS}

educational games, Twitch, live streaming

\section{ACM Reference Format:}

Noor Hammad, Erik Harpstead, and Jessica Hammer. 2021. Towards Examining The Effects of Live Streaming an Educational Game. In CHI Conference on Human Factors in Computing Systems Extended Abstracts (CHI '21 Extended Abstracts), May 8-13, 2021, Yokohama, Japan. ACM, New York, NY, USA, 6 pages. https://doi.org/10.1145/3411763.3451616

\section{INTRODUCTION}

Online learning has become ever-present in the education of students at all levels. Educational technologies have enabled learners to have access to massive amounts of educational content. While asynchronous content such as YouTube videos have democratized access to education, and have been shown to support observational

(C)
This work is licensed under a Creative Commons Attribution International
4.0 License.
CHI '21 Extended Abstracts, May 8-13, 2021, Yokohama, Japan
(C) 2021 Copyright held by the owner/author(s).
ACM ISBN 978-1-4503-8095-9/21/05.
https://doi.org/10.1145/3411763.3451616

learning, there are limited participation opportunities in the learning activity.

The disruption of education caused by the COVID-19 pandemic, which has seen the replacement of in-class learning with remote instruction, further exposed the participatory shortcomings in online learning. The combination of video calls and asynchronous activities has led to an isolated learner experience, which has contributed to what has been labelled as "Zoom fatigue" among learners and working professionals alike [42]. Lines of literature in the learning sciences suggests that more active and interactive forms of learning are better than observational learning alone [11], and there is a greater call for more interactive online learning experiences. Live streaming platforms, such as Twitch.tv [3] and YouTube Live [4], present an opportunity to support participatory learning.

Unlike asynchronous video viewing, live streaming platforms allow streamers to share footage in real time with live audiences $[17,37]$. A viewer typically sees the streamer's footage along with a camera view of the streamers themselves. Interaction is supported through text chat where they can discuss with other viewers or connect with the streamer. Opportunities to extend streaming interfaces are also being explored through video overlays and audience participation games [24, 38]. Despite the saturation of video-based engagement as a result of the pandemic, live stream platforms are experiencing an exponential growth in popularity. Over one billion hours of content was watched on Twitch in December 2020 alone $[2,40]$, exemplifying the need for more participatory viewing experiences, and highlighting the potential of live streaming as an educational medium.

The use of live streaming for learning is a growing subject of study in the HCI research community. While live streaming platforms were not initially built with learning in mind, research has shown that learning is already happening on live stream platforms, and has even been adopted by instructors during the pandemic [8]. Some viewers of game streams, which are a large proportion of live stream content, are motivated to engage in order to improve their own play [25], while learning communities surrounding topics such as software development and other creative streams can also be found $[18,21]$. Much is still unknown about how formal and informal learning can be supported with existing live streaming interfaces and what kinds of educational experiences best leverage the strengths of live streaming. Our work is an instance exploring how learning can manifest through live streamed content. In particular, we are exploring the learning effects of streaming educational games.

Educational games have been used in classrooms to teach domainspecific knowledge through interactive play. Unlike entertainment games, educational games are built with certain learning outcomes 
in mind. While previous work has shown that viewers are motivated to engage in entertainment game streams for learning purposes, and that it is possible to improve in-game performance from watching live streams of games [36], there is little research on utilizing live streams to transfer domain knowledge as opposed to enhancing in-game skill alone.

The growing popularity of live streaming platforms and demand for novel online learning experiences have motivated us to explore the potential of educational games as a form of a participatory, live streamed learning experience. In this paper, we share our inprogress work examining how live streaming an educational game can facilitate learning gains. We share our plan for a mixed methods study comparing the learning gains from a live streamed educational game called Angle fungle [28] to other forms of instruction. We will compare how streaming the game compares to playing the game, watching the game without interaction enabled, and a remote lecture covering the same angle-related concepts. When complete, our study will contribute an understanding of how existing live streaming platforms support, or fail to support, observational learning from educational games, creating further opportunities to create novel, live streamed learning experiences.

\section{BACKGROUND}

Our work looking to examine the learning effects of streaming an educational game draws on multiple bodies of literature. In particular, we draw from previous work on learning from live streaming, educational games and the importance of interaction from the perspective of the ICAP theory.

\subsection{Learning From Live Streaming}

The streaming interface, which includes a stream of the content played by the streamer, a camera view of the streamer themselves, and a chat window where they can connect with viewers, is largely ubiquitous across live stream platforms [37]. Twitch is by far the most popular live streaming platform, with a large proportion of content focusing on games. It is a freely accessible platform, and streamers can use open-source broadcasting software to stream gameplay on mobile, console, or computer to their Twitch channel [1]. Multiple studies have examined viewer motivations on Twitch, revealing how community engagement and social interaction play a major role in the viewer experience compared to other mass media $[25,39]$.

In the formal learning context, recent work by Chen et. al has examined the challenges and prospects instructors in China have faced when adopting live streaming during the COVID-19 pandemic [8]. Lu et. al examined the use of live streaming in China for intangible cultural heritage ( $\mathrm{ICH})$, revealing that streamers are altruistically motivated to stream their work [35]. Studies by Faas et. al and Fraser et. al have examined informal learning communities on Twitch $[18,21]$. Their work has shown that viewers are motivated by learning and inspiration, and that these live stream communities can foster better learning experiences for viewers through streamer interaction along with co-mentoring among the streamer and their viewers. Further studies have been conducted studying programming live streams [5, 9]. The works cited above have also acknowledged the shortcomings in live stream platforms, such as the limitations of text chat, difficulty for drop-in viewers and streamers' struggles to balance engagement and streaming the content. These shortcomings are partly due to live streaming platforms not being built with learning in mind [9]. This requires future research to design better live streaming interfaces for learning, yet also to better grasp what benefits there are to utilizing live streaming for various types of educational content.

Learning has also been shown to be possible in game streams, which still makes up a large portion of live streamed content [2]. Payne et. al's study of the worked example effect's presence on Twitch has shown that increased in-game performance can be possible when viewers engage in a live stream under certain conditions [36]. Their work revealed that learning from novice streamers is as good as that of experts, and that learning gains for extroverts occur when given the opportunity to interact with other learners through the chat. Payne et. al's examination was centered around League of Legends and the completion of an in-game task. Payne et. al's study of how live streaming can affect in-game performance is a major inspiration for our work here; rather than examining how in-game performance can be enhanced for expertise in an entertainment game such as League of Legends, we seek to understand how the viewing of an educational game can enhance the expertise in the learning outcomes the educational game targets.

\subsection{Learning From Educational Games}

Educational games are increasingly used in classrooms and have become a popular form of educational technology, with nearly $74 \%$ of K-8 teachers in the United States making use of educational games for instruction as of 2014 [41]. While systematic reviews of learning from games have yielded mixed results $[12,14]$, teachers who regularly use games in their classrooms reported greater improvement in their students' skills [41].

At their core, educational games are experiences built from the ground up to support learning through playing [22]. They can give players opportunities to learn by "doing" in the game's context, but where the player still has the freedom to fail [22, 29]. Games also provide opportunities for embedded assessment, where players' learning is evaluated within the game itself [33]. Compared to other active learning activities, educational games typically provide better cognitive outcomes, but only some studies found differences in motivation. These meta-analyses do find, though, that games can produce learning under the right circumstances. For example, games and learning activities must be aligned with one another so that learners do not spend time on irrelevant activities [6], but not over-scaffold player behavior [34].

In their studies examining the influence of intrinsic motivation on learning gains and time on task in the mathematics game, Zombie Division, Habgood et. al revealed that participants experienced greater learning gains and played seven times longer than those in the extrinsic and control variants [23]. This indicates that, should students find a game intrinsically motivating, they will experience greater value from the experience. Creating the right circumstances for game-based learning involves designing the learning experience as carefully as the game. Moreover, intrinsic motivation cannot be mandated; When games become mandatory, they also become less engaging [27]. This finding creates challenges when deploying 
games in classrooms and other institutional environments, further adding to existing deployment issues such as cost and alignment with curriculum [16].

A key aspect on which the success of an educational game hinges on is the learner's ability to transfer the in-game context into domain knowledge where it can be useful [26]. For example, many game-based learning experiences include a post-game reflective process in which participants make sense of their experiences [15, 19], which encourages the knowledge transfer.

\subsection{The Importance of Interactivity in Learning}

Many learning theorists recommend learning by doing activities, rather than through watching videos or reading text [7]. By actively practicing skills in context, learners can better understand what prior knowledge to bring to bear [43]; they can receive rapid feedback on their progress and experiment with different strategies [20]; and they can seek out as-needed instruction within the context of the activity [13, 32]. In this regard, interaction in the learning activity is key to provide rapid feedback on learner progress. For example, in the context of MOOCs (massively open online courses), Koedinger et. al. demonstrated that interactive activities outperformed watching course videos by a factor of six [30], showing a causal link between interactive activities and learning [31].

A model that inspires our work is known as the ICAP framework [11]. ICAP is an acronym for Interactive, Constructive, Active, and Passive. Created by Chi and Wyie, the framework provides a model on which effective learning depends on the level of interactivity. It proposes that interactive learning activities, in which multiple learners co-create learning-relevant material, are better for learning than constructive activities, which involve generative behaviors alone. Both these approaches are better than active learning, where learners manipulate but do not generate learning materials, which in turn is better than passive learning marked by simply paying attention. This hierarchy of learning highlights how live streaming's greater avenues for interaction with the learning activity may be more effective than videos alone. However, implementing the ICAP framework in the design of learning activities can be challenging. For example, K-12 instructors struggled to generate interactive learning activities for the classroom [10].

The ICAP framework emphasizes interactivity in the learning process. Live streaming, with its support of real-time interaction between streamers and viewers, has shown promise for learningoriented content and communities, which have so far not made any significant use of educational games. With this work, we aim to test how the learning outcomes of an educational game translate to the live streaming context.

\section{PROPOSED METHODOLOGY}

To begin understanding the potential of live streaming platforms for educational games, we formulated a study centered around the following research questions:

(1) How do the learning outcomes of an educational game transfer to a live streaming context?

(2) What role does interactivity in live streaming, namely the text chat function, play in the learning experience?

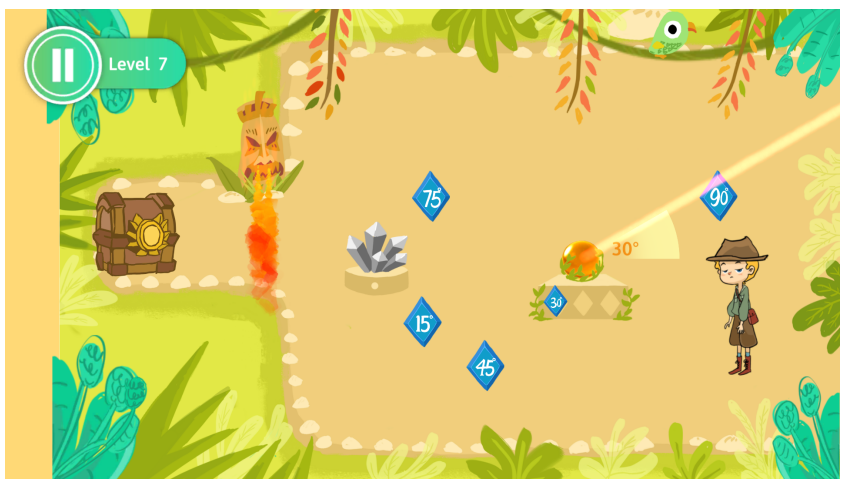

Figure 1: A level in Angle Jungle. The player must drag the blue diamonds in order to move the beam, which will clear a path to the treasure

Using these questions, our planned study will involve streaming an educational game to the game's target demographic and comparing the learning outcomes against the original, non-streamed version of the game and other conditions described below.

\subsection{Setting and Participants}

The study will include participants from a local school. Participants will be students in fourth to sixth grade. All students will have the opportunity to play the game at the end of the study. We will log the participant's age and grade in our data collection to determine how grade level affected learning outcomes in our analysis.

To address privacy and data concerns involving young children on public live streams, we will use the webinar feature of the video conferencing software Zoom as our live streaming platform. This is Zoom's live streaming feature with an interface that is akin to platforms like Twitch and Youtube, while allowing us to provide a more controlled private environment that does not share data outside of our team. Zoom Webinars are regularly used for live streaming events, and our participants are familiar with the Zoom interface. Instructors from the school will be present throughout the study to help set up the study and make them feel more comfortable.

\subsection{Angle Jungle}

The game we will use for our study is called Angle fungle [28]. Angle fungle is an award-winning educational game that teaches angle representation concepts to students in fourth to sixth grade. It is an iOS single-player puzzle game consisting of thirty levels wherein the player must use angles to overcome obstacles and reach the treasure (see Figure 1). Players place gems containing the numeric representation of an angle onto a "beam maker" that moves a light beam according to the inserted gem. To clear the path to the treasure, the beam is manipulated using inserted angle gems to hit a target.

Angle fungle was heuristically evaluated with teachers to determine its effectiveness. In addition to reinforcing numeric-spatial learning, Angle fungle's teaches positive and negative angle representation, clockwise and counterclockwise rotation, and protractor 
use; concepts that local teachers reported as being a source of confusion for students [28] One shortcoming of using Angle fungle is that it targets a younger audience that may not be comfortable with interacting through chat in a live stream.

To further improve the ease of access, we deployed a web version of the game for the study, modifying its touch controls to be mousebased in the process. This is particularly critical for the execution of the study given the pandemic; deploying the game on the web means we do not need to distribute iOS devices to our participants.

\subsection{Conditions}

In order to test the research questions we established, we formulated a study design that lets us examine differences in non-game domain learning under different educational game and streaming designs.The study consists of four mutually exclusive conditions:

3.3.1 The Live Stream Condition: Participants in this condition will join a Zoom webinar of a streamer playing Angle fungle. As is commonplace in live streams, the participants will have access to the text chat, and the streamer will actively engage and interact with the participants. The streamer will be a member of the research team that is trained in Twitch streaming. To ensure consistency during the session, we created a set of guidelines for the streamer to follow as they make their way through Angle fungle to better align with modern streaming personas and help simulate the live streaming experience. Key guidelines include: attentively monitor the text chat; practice think-aloud behavior and provide commentary during gameplay; cultivate a two-way conversation with the audience by responding to their requests, suggestions, and questions; maintain a respectful and inclusive environment for all participants.

3.3.2 The Play Condition: Here, participants play a non-live streamed version of Angle fungle as it was originally meant to be played. Participants in this condition will play Angle fungle individually through a web browser, and are given the freedom to complete levels and learn the mechanics of the game.

3.3.3 The Viewing Condition: This condition is a non-interactive viewing condition that simulates an asynchronous video experience to examine the gains from observational learning alone. Unlike the live stream condition where participants watch and interact with a streamer play Angle fungle in real-time, participants will instead watch the game without text chat interaction enabled. This condition captures the asynchronous video viewing experience, however it will still take place in the Zoom webinar for consistency. To further maintain consistency, the game will still be played by the same streamer from the live stream condition, however the guidelines they will follow do not include the focus on interactivity with the audience. We added this condition to understand the role observational learning plays within the context of Angle fungle. By comparing this condition with the live stream condition, we can better understand the role that the live interaction plays in the learning process.

3.3.4 Virtual Instruction: This condition does not involve Angle fungle. Establishing a baseline to compare with the educational game conditions helps us better understand Angle fungle's role in the learning process, and the unprecedented move to online instruction has made the understanding from this condition even more critical. The participants will take part in a remote lesson via Zoom covering the angle related concepts that Angle fungle aims to teach. The instructor will be the same streamer from previous conditions. While we considered utilizing the participants' usual instructors in this condition to capitalize on the participants' familiarity with their teachers, we chose to maintain consistency at the expense of this established rapport. This decision is also influenced by our use of students across three grades in each condition; there are different instructors for each grade and thus no single instructor could have been chosen. Under normal circumstances, this condition would have been executed in a classroom setting, however our participants are now familiar with the online learning format and receiving instruction through Zoom.

The above conditions will give us preliminary understanding of the effect live streaming an educational game has on learning, and how interaction affordances of the live streaming interface is involved in that effect. Other variable manipulations and conditions were considered, such as streamer expertise [36] and live streaming interface variations. As this is an initial investigation into the space, we chose to limit the condition as we did to build a foundation from which future study conditions can build from.

\subsection{Measures and Procedure}

Our primary measure of the learning gains will be in the form of a pre and post test focusing on angle concepts. Both tests will have five questions, and participants are given twenty minutes to complete them. Questions range from ranking angles from smallest to largest, estimating angle measure, and addition and subtraction of angles. The questions are taken directly from the local curriculum standard, and were reviewed by the head mathematics instructor at our partner school. Along with the tests, we will supplement the quantitative data by also recording all video, audio and chat data for each condition. While the tests are used to determine the degree of learning gains, we will use the remainder of the data set to examine emergent phenomena from our study and what behaviors emerged in each condition. The video and audio data in particular will be used to code how closely the streamer followed the guidelines we created.

The study was designed to take approximately one hour of the participants' time. The procedure of our study will be as follows:

(1) Participants, with the help of their instructors, are assigned an anonymous identifier that they will use throughout the study

(2) Participants complete the digital pre-test. They are given twenty minutes to do so.

(3) Participants are randomly assigned to one of the four conditions, and change their display name in their assigned Zoom room. All study activity will be done using the computers provided to the participants by the school.

(4) Participants engage in the learning activity from their condition, meaning they will either:

- Play Angle fungle through a web browser for twenty minutes

- Engage in a live stream of Angle fungle for twenty minutes 
- Watch a video of Angle fungle for twenty minutes

- Take part in a virtual lesson on angles for twenty minutes

(5) Participants complete the post-test. They are given twenty minutes to do so

At the end of the study, participants in all conditions are given the opportunity to play Angle fungle.

\subsection{Anticipated Observations}

Our proposed study will be the first to examine how live streaming an educational game affects learning outcomes, however we anticipate the following outcomes:

(1) Learning gains from the stream condition will be greater than the viewing condition, but less than the play condition. This is because viewing is a more passive form of learning than with interaction enabled, which limits the participants' ability to ask questions. Given the game was originally meant to be played without live streaming, and that playing the game results in a more individualized learning experience, it is thus highly likely that playing the game will be more effective than the stream conditions.

(2) Learning gains from the virtual instruction condition will be greater than all other conditions. This is because participants are now used to virtual instruction via Zoom, and because Angle fungle was not built for live streaming interfaces.

\section{FUTURE WORK}

Upon executing the study, we will analyze the data to determine the learning gains from our four conditions. From these findings, we will design future studies focusing on the finer details of the effects of educational game streaming. Future avenues include examining the effect that different streamer personas and expertise levels have on the learning gains from educational game streaming, and qualitative interviews and ethnographies to better understand the motivations (or lack thereof) of streamers and viewers in engaging with educational games on live stream platforms.

Our primary motivation for pursuing this work is to create novel educational experiences, and to make educational games more accessible using live streaming platforms. Live streaming games is an affordable option to bring educational games back into the classroom. However, most educational games were not built for live streaming interfaces, and live streaming platforms themselves were not built with learning and education in mind. We seek to understand how learning happens as is from these platforms so as to improve current interfaces, leveraging their strengths and mitigating weaknesses to broaden access to educational games.

The emergence of audience participation games on Twitch bring forth a promising avenue of future work to improve live streaming for education. Our current phase of work is concerned with understanding the current state of live streaming interfaces; the next phase will focus on the design of novel interfaces and audience participation games that can push the potential of live streaming as an educational platform forward.

\section{ACKNOWLEDGMENTS}

We would like to thank Gail Kusbit for her incredible support throughout this process. We are grateful to our reviewers for their constructive feedback. This work is funded by the National Science Foundation (NSF) Grant Number 1942087.

\section{REFERENCES}

[1] 2021. Open Broadcast Software. https://obsproject.com/

[2] 2021. TwitchTracker Statistics and Charts. https://twitchtracker.com/statistics

[3] 2021. Twitch.tv. https://www.twitch.tv/

[4] 2021. Youtube. https://www.youtube.com/

[5] Abdulaziz Alaboudi and Thomas D LaToza. 2019. An Exploratory Study of Live-Streamed Programming. In 2019 IEEE Symposium on Visual Languages and Human-Centric Computing (VL/HCC). IEEE, 5-13.

[6] Vincent Aleven, Eben Myers, Matthew Easterday, and Amy Ogan. 2010. Toward a framework for the analysis and design of educational games. In 2010 third IEEE international conference on digital game and intelligent toy enhanced learning. IEEE, 69-76.

[7] Yuichiro Anzai and Herbert A Simon. 1979. The theory of learning by doing. Psychological review 86, 2 (1979), 124

[8] Xinyue Chen, Si Chen, Xu Wang, and Yun Huang. 2021. "I was afraid, but now I enjoy being a streamer!" Understanding the Challenges and Prospects of Using Live Streaming for Online Education. Proceedings of the ACM on Human-Computer Interaction 4, CSCW3 (2021), 1-32.

[9] Yan Chen, Walter S Lasecki, and Tao Dong. 2021. Towards Supporting Programming Education at Scale via Live Streaming. Proceedings of the ACM on Human-Computer Interaction 4, CSCW3 (2021), 1-19.

[10] Michelene TH Chi, Joshua Adams, Emily B Bogusch, Christiana Bruchok, Seokmin Kang, Matthew Lancaster, Roy Levy, Na Li, Katherine L McEldoon, Glenda S Stump, et al. 2018. Translating the ICAP theory of cognitive engagement into practice. Cognitive science 42, 6 (2018), 1777-1832.

[11] Michelene TH Chi and Ruth Wylie. 2014. The ICAP framework: Linking cognitive engagement to active learning outcomes. Educational psychologist 49, 4 (2014), 219-243.

[12] Douglas B Clark, Emily E Tanner-Smith, and Stephen S Killingsworth. 2016. Digital games, design, and learning: A systematic review and meta-analysis. Review of educational research 86, 1 (2016), 79-122.

[13] Allan Collins. 1991. Cognitive apprenticeship and instructional technology. Educational values and cognitive instruction: Implications for reform 1991 (1991), 121-138.

[14] Thomas M Connolly, Elizabeth A Boyle, Ewan MacArthur, Thomas Hainey, and James M Boyle. 2012. A systematic literature review of empirical evidence on computer games and serious games. Computers \& education 59, 2 (2012), 661-686.

[15] David Crookall. 2014. Engaging (in) gameplay and (in) debriefing.

[16] Frederik De Grove, Jeroen Bourgonjon, and Jan Van Looy. 2012. Digital games in the classroom? A contextual approach to teachers' adoption intention of digital games in formal education. Computers in Human behavior 28, 6 (2012), 2023-2033.

[17] Jie Deng, Felix Cuadrado, Gareth Tyson, and Steve Uhlig. 2015. Behind the game: Exploring the twitch streaming platform. In 2015 International Workshop on Network and Systems Support for Games (NetGames). IEEE, 1-6.

[18] Travis Faas, Lynn Dombrowski, Alyson Young, and Andrew D Miller. 2018. Watch me code: Programming mentorship communities on twitch. tv. Proceedings of the ACM on Human-Computer Interaction 2, CSCW (2018), 1-18.

[19] Ruth M Fanning and David M Gaba. 2007. The role of debriefing in simulationbased learning. Simulation in healthcare 2, 2 (2007), 115-125.

[20] Justin Fanzo, Rachel Gu, Jinchao Han, Ketul Majmudar, Tony Deng, Flora Cheng, Chaoya Li, and Jessica Hammer. 2017. What Lurks in the Dark: An Audience Participation Horror Game. In Extended Abstracts Publication of the Annual Symposium on Computer-Human Interaction in Play. 621-624.

[21] C Ailie Fraser, Joy O Kim, Alison Thornsberry, Scott Klemmer, and Mira Dontcheva. 2019. Sharing the studio: How creative livestreaming can inspire, educate, and engage. In Proceedings of the 2019 on Creativity and Cognition. 144-155.

[22] James Paul Gee. 2003. What video games have to teach us about learning and literacy. Computers in Entertainment (CIE) 1, 1 (2003), 20-20.

[23] MP Jacob Habgood and Shaaron E Ainsworth. 2011. Motivating children to learn effectively: Exploring the value of intrinsic integration in educational games. The fournal of the Learning Sciences 20, 2 (2011), 169-206.

[24] Emily Halpin. [n.d.]. Introducing Extensions: A Streaming Revolution. https://blog.twitch.tv/en/2017/08/31/introducing-extensions-a-streamingrevolution-c31762addcd9/

[25] William A Hamilton, Oliver Garretson, and Andruid Kerne. 2014. Streaming on twitch: fostering participatory communities of play within live mixed media. In Proceedings of the SIGCHI conference on human factors in computing systems. $1315-1324$.

[26] Jessica Hammer and John Black. 2009. Games and (preparation for future) learning. Educational Technology (2009), 29-34.

[27] Carrie Heeter, Yu-Hao Lee, Brian Magerko, and Ben Medler. 2011. Impacts of forced serious game play on vulnerable subgroups. International fournal of 
Gaming and Computer-Mediated Simulations (IfGCMS) 3, 3 (2011), 34-53.

[28] Jibran Khan, Jun Wang, Xueyang Wang, Yonglin Zhang, Jessica Hammer, Scott Stevens, and Ricardo Washington. 2017. Angle Jungle: an educational game about angles. In Extended Abstracts Publication of the Annual Symposium on Computer-Human Interaction in Play. 633-638.

[29] Eric Klopfer, Scot Osterweil, Katie Salen, et al. 2009. Moving learning games forward. Cambridge, MA: The Education Arcade (2009).

[30] Kenneth R Koedinger, Jihee Kim, Julianna Zhuxin Jia, Elizabeth A McLaughlin, and Norman L Bier. 2015. Learning is not a spectator sport: Doing is better than watching for learning from a MOOC. In Proceedings of the second (2015) ACM conference on learning@scale.111-120.

[31] Kenneth R Koedinger, Elizabeth A McLaughlin, Julianna Zhuxin Jia, and Norman L Bier. 2016. Is the doer effect a causal relationship? How can we tell and why it's important. In Proceedings of the Sixth International Conference on Learning Analytics \& Knowledge. 388-397.

[32] Jean Lave, Etienne Wenger, et al. 1991. Situated learning: Legitimate peripheral participation. Cambridge university press.

[33] Christian Sebastian Loh, Yanyan Sheng, and Dirk Ifenthaler. 2015. Serious games analytics. Edited by Christian Sebastian Loh, Yanyan Sheng, and Dirk Ifenthaler Cham: Springer International Publishing. doi 10 (2015), 978-3.

[34] Yanjin Long and Vincent Aleven. 2017. Educational game and intelligent tutoring system: A classroom study and comparative design analysis. ACM Transactions on Computer-Human Interaction (TOCHI) 24, 3 (2017), 1-27.

[35] Zhicong Lu, Michelle Annett, Mingming Fan, and Daniel Wigdor. 2019. "I feel it is my responsibility to stream" Streaming and Engaging with Intangible Cultura Heritage through Livestreaming. In Proceedings of the 2019 CHI Conference on
Human Factors in Computing Systems. 1-14.

[36] Katherine Payne, Mark J Keith, Ryan M Schuetzler, and Justin Scott Giboney. 2017. Examining the learning effects of live streaming video game instruction over Twitch. Computers in Human Behavior 77 (2017), 95-109.

[37] Karine Pires and Gwendal Simon. 2015. YouTube live and Twitch: a tour of user-generated live streaming systems. In Proceedings of the 6th ACM multimedia systems conference. $225-230$.

[38] Joseph Seering, Saiph Savage, Michael Eagle, Joshua Churchin, Rachel Moeller, Jeffrey P Bigham, and Jessica Hammer. 2017. Audience participation games: Blurring the line between player and spectator. In Proceedings of the 2017 Conference on Designing Interactive Systems. 429-440.

[39] Max Sjöblom and Juho Hamari. 2017. Why do people watch others play video games? An empirical study on the motivations of Twitch users. Computers in human behavior 75 (2017), 985-996.

[40] Bijan Stephen. 2020. 2020 was the year of the Twitch streamer. https://www.theverge.com/2020/12/16/22167590/2020-the-year-of-thetwitch-streamer-webcam-microphone

[41] Lori M Takeuchi and Sarah Vaala. 2014. Level up Learning: A National Survey on Teaching with Digital Games.. In foan Ganz Cooney Center at Sesame Workshop. ERIC.

[42] Michael Wong. 2020. Managing Zoom Fatigue. https://tlconestoga.ca/zoomfatigue/

[43] Xinming Zhu, Yifei Lee, Herbert A Simon, and Dan Zhu. 1996. Cue recognition and cue elaboration in learning from examples. Proceedings of the National Academy of Sciences 93, 3 (1996), 1346-1351. 